\title{
Birth of the nanolaser
}

\author{
Laser science looks set to reach new dimensions, following the use of plasmonics to confine optical energy \\ in laser nanocavities, and the recent claims of blue and green stimulated emission from two such lasers.
}

Ever since the laser was first demonstrated in 1960 (get ready for some serious 50th birthday celebrations next year), scientists have strived to find ways of making lasers smaller and more convenient to use. We've certainly come a long way from the first table-top gas and crystal lasers to the tiny semiconductor laser diodes that are prevalent today. The question of how small a laser can actually be is an interesting one. For many years it's been thought that the ultimate limit is constrained by the need to fit an optical mode inside a cavity; the consequence being that diffraction is the key limit and therefore a laser cavity cannot be smaller than about half the wavelength of the emitted light. Indeed, in the past few years many intriguing designs of microscopic lasers based on tiny pillars $^{1}$, nanowires ${ }^{2}$ and photonic crystals ${ }^{3}$ have all approached this limit but not exceeded it.

Now, as reported by two experimental papers appearing at almost the same time in Nature, it seems that this limit has been surpassed and that nanoscale lasers with cavity dimensions well below the wavelength of light are within reach. The papers in question, one from the team of Noginov et al. ${ }^{4}$ in a collaboration between Purdue, Norfolk State, and Cornell Universities, and the other from Oulton et al. ${ }^{5}$ in Berkeley, California, both rely on the science of plasmonics to get around the usual cavity size limit. The clever idea behind both the papers is that instead of confining optical energy in a cavity in the form of a conventional optical mode, it is confined with the help of surface-plasmons - free-electron oscillations that are bound to the interface between a metal and a dielectric — which can be far smaller. The great attraction of plasmons is not only that they can store optical energy on a very small dimension (Oulton et al. quote mode areas of $\lambda^{2} / 400$ ) but that they are easy to excite by light and convert back into light.

As in a conventional laser cavity, the idea is that losses can be overcome by adding some form of optical gain, thus producing stimulated amplification and lasing that is then outcoupled from the cavity in the form of light. If sufficient gain can be

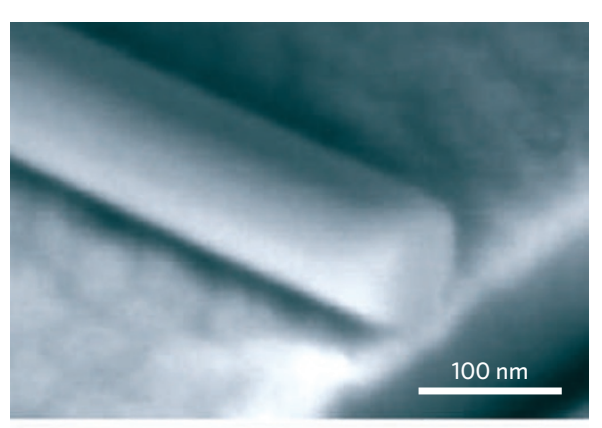

with 405-nm light, stimulated emission was observed at $489 \mathrm{~nm}$ (blue).

Although these reports of green and blue nanolasers are exciting, the big challenge now is finding ways to electrically, rather than optically, pump such devices. The other task for future research is finding convenient ways to modulate such lasers at high bit-rates, without dramatically increasing their footprint or power consumption. If solutions to these issues are found, this research offers the tantalizing prospect of having electrically driven nanolasers on the scale of state-of-theart electronics, thereby hinting at the prospect of incredibly dense optoelectronic integrated circuitry. Other potential applications that may benefit from such nanolasers are miniature biosensors and the field of quantum information processing.

Similarly, the exciting progress being made in excitonic devices also gives great hope for the creation of a new breed of dense and powerful optoelectronic circuitry. Just like plasmons, excitons (bound electronhole pairs that form in semiconductors) can be easily excited and converted into light, offering exciting new prospects for making highly compact and sophisticated optoelectronic circuitry that goes beyond what is possible in purely optical or electronic domains. On page 577 of this issue ${ }^{6}$, an excitonic optical switch operating at a new temperature limit of $125 \mathrm{~K}$ is reported, and although it is early days, the field seems to be maturing rapidly.

It is clear that both plasmonics and excitonics are still in their infancy, but it will be fascinating to watch both technologies evolve in coming years. Perhaps a new era of indirect manipulation of light in metals is now truly upon us, with semiconductor and dielectric nanostructures circumnavigating the conventional limitations of photonics. These are exciting times indeed.

\section{References}

1. Hill, M. T. et al. Nature Photon. 1, 589-594 (2007).

2. Johnson, J. C. et al. Nature Mater. 1, 106-110 (2002).

3. Altug, H., Englund, D. and Vučkovič, J. Nature Phys. 2, 484-488 (2006).

4. Noginov, M. A. et al. Nature 460, 1110-1112 (2009).

5. Oulton, R. F. et al. Nature doi:10.1038/nature08364 (2009).

6. Grosso, G. et al. Nature Photon. 3, 577-580 (2009). 